\title{
A spectral route to determining chirality
}

\author{
Pedersen, Jesper Goor; Mortensen, Asger
}

Published in:

Applied Physics Letters

Link to article, DOI:

$10.1063 / 1.3246153$

Publication date:

2009

Document Version

Publisher's PDF, also known as Version of record

Link back to DTU Orbit

Citation (APA):

Pedersen, J. G., \& Mortensen, A. (2009). A spectral route to determining chirality. Applied Physics Letters, 95(15), 151104. https://doi.org/10.1063/1.3246153

\section{General rights}

Copyright and moral rights for the publications made accessible in the public portal are retained by the authors and/or other copyright owners and it is a condition of accessing publications that users recognise and abide by the legal requirements associated with these rights.

- Users may download and print one copy of any publication from the public portal for the purpose of private study or research.

- You may not further distribute the material or use it for any profit-making activity or commercial gain

- You may freely distribute the URL identifying the publication in the public portal

If you believe that this document breaches copyright please contact us providing details, and we will remove access to the work immediately and investigate your claim. 


\title{
A spectral route to determining chirality
}

\author{
Jesper Goor Pedersen ${ }^{\mathrm{a})}$ and Niels Asger Mortensen \\ Department of Photonics Engineering, DTU Fotonik, Technical University of Denmark, Building 343, \\ DK-2800 Kongens Lyngby, Denmark
}

(Received 30 June 2009; accepted 20 September 2009; published online 13 October 2009)

\begin{abstract}
We show how one-dimensional structured media can be used to measure chirality, via the spectral shift of the photonic band gap edges. Analytically, we show that a chiral contrast can, in some cases, be mapped unto an index contrast, thereby greatly simplifying the analysis of such structures. Using this mapping, we derive a first-order shift of the band gap edges with chirality. Potentially, this effect could be used for measuring enantiomeric excess. () 2009 American Institute of Physics.
\end{abstract}

[doi:10.1063/1.3246153]

The concept of molecular enantiomers, molecules which are nonsuperposable mirror images of each other, is of vital importance in the pharmaceutical industry, due to the fact that two different stereoisomers of the same molecule may have profoundly different effects. Such chiral molecules have particular optical properties, such as, e.g., optical activity, and circular dichroism. ${ }^{1}$ Also, as was recently shown experimentally, linearly polarized light incident upon a chiral medium will split into the two directions of circular polarization, refracted at different angles to each other. ${ }^{2}$ Such properties may be utilized to determine the chirality of a given sample, a measurement also of the enantiomeric excess. However, neither of these methods are based on spectral information but instead rely on measurements of intensity or angle of refraction. While both circular dichroism and optical rotation are of course wavelength dependent, this wavelength dependence is not what is used to characterize the chiral strength in such measurements. We have previously shown how the sensitivity of measurements based on circular dichroism may be enhanced using photonic crystals. ${ }^{3}$ In this letter, we present a possible way of obtaining spectral signatures of chirality, by tracking the band gap edges of such structured media.

We consider a Bragg stack, as illustrated in Fig. 1, consisting of alternating layers of isotropic chiral media, characterized by refractive indices $n_{i}$, and chiral parameters $\gamma_{i}$, with $i=1,2$. The layers are of widths $d_{i}$, with lattice constant $\Lambda$ $=d_{1}+d_{2}$. Such structures are closely related to magnetooptical Bragg gratings, in which the optical activity stems from the inclusion of magnetic material. ${ }^{4}$ To treat the inclusion of the chiral media, we need to modify the ordinary constitutive relations. We assume, as is commonly done, that the curl equations of Maxwell still hold in chiral media, but replace the standard constitutive relations with the ones based on the symmetrized Condon set, ${ }^{5,6}$

$$
\mathbf{D}=\epsilon \mathbf{E}-g \partial \mathbf{H} / \partial t, \quad \mathbf{B}=\mu \mathbf{H}+g \partial \mathbf{E} / \partial t,
$$

which for monochromatic plane waves with time dependence $\exp (-i \omega t)$ becomes

$$
\mathbf{D}=\epsilon \mathbf{E}+i \gamma \mathbf{H}, \quad \mathbf{B}=\mu \mathbf{H}-i \gamma \mathbf{E},
$$

where $\gamma=\omega g$. For weak chirality, these constitutive relations are equivalent to the Drude-Born-Fedorov relations, as dis-

${ }^{a)}$ Electronic mail: jeped@fotonik.dtu.dk. cussed in the review by Lekner in Ref. 7. We implicitly assume that $\epsilon$ and $\gamma$ may be position dependent, while we for simplicity consider nonmagnetic materials and set $\mu=1$ throughout the paper. We calculate the dispersion relation of the chiral structure using a recent derivation by Kim et al., ${ }^{8}$ which is based on an exact solution of the coupled wave equations of the system.

In Fig. 2 we show the band structure of a Bragg stack in which one of the constituent media is chiral. For illustrative purposes, we choose a value of $\gamma=0.6$ for the chiral medium, which is several orders of magnitude larger than any naturally occurring media. We stress that the trends observed remain the same for more realistic values of the chiral strength, and that none of the conclusions drawn depend on this magnitude of chirality. For reference we also show the band structure for the corresponding nonchiral structure. The results are shown for on-axis and oblique incidence of light, respectively. In both cases the band structure is significantly altered as chirality is introduced in one of the media. However, the location and the size of the band gaps is unaltered in the case of on-axis incidence. On the other hand, it is clear that for oblique incidence, there is a marked shift of the band gap edges as a chiral medium is introduced. This suggests a possible spectral route to measuring chirality, namely that the chirality may be determined by tracking the band gap edges of the Bragg stack. One could imagine having a Bragg stack consisting of air and ordinary, nonchiral dielectric and then flowing a chiral liquid through the air regions. By measuring transmission spectra it would then be possible to determine the chirality of liquid.

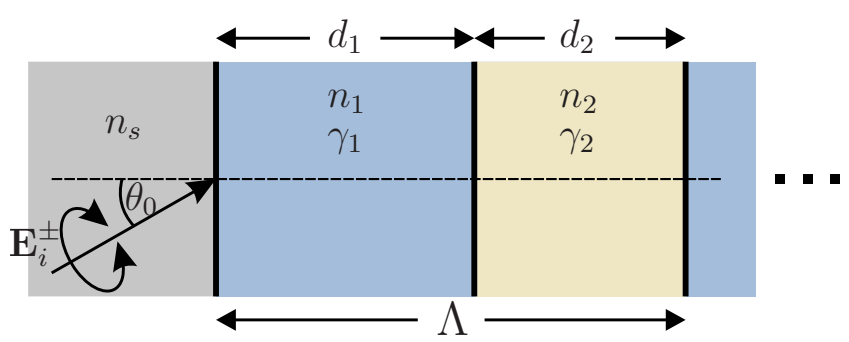

FIG. 1. (Color online) The chiral Bragg stack consisting of alternating layers of refractive indices $n_{1}$ and $n_{2}$, and chiral strengths $\gamma_{1}$ and $\gamma_{2}$. The widths of the layers are $d_{1}$ and $d_{2}$, so the lattice constant $\Lambda=d_{1}+d_{2}$. Circularly polarized light is incident at an angle $\theta_{0}$ from the surrounding medium with refractive index $n_{s}=1$. 

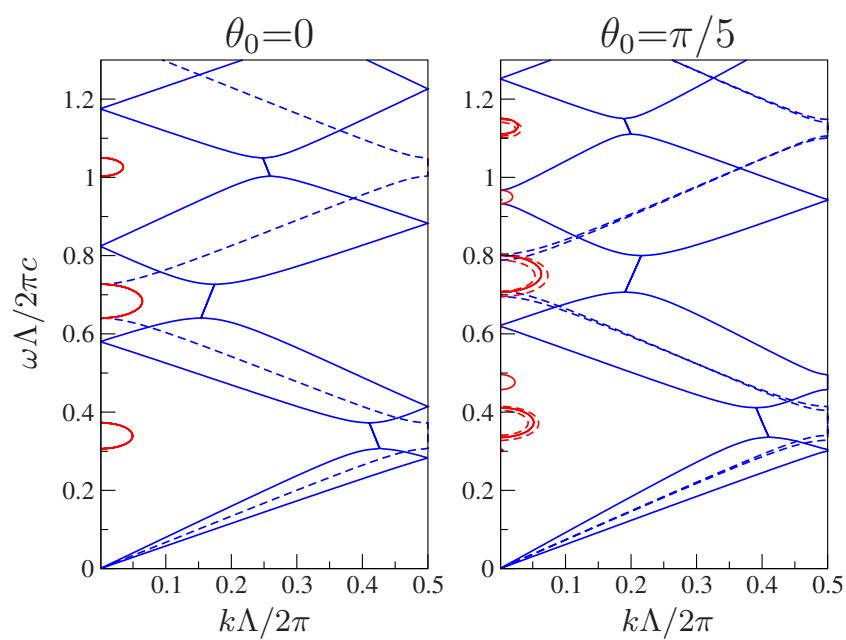

FIG. 2. (Color online) Band structures for a chiral Bragg stack with layers of thickness $d_{1}=0.2 \Lambda$ and $d_{2}=0.8 \Lambda$, refractive indices $n_{1}=2$ and $n_{2}=1.33$, and $\gamma_{1}=0$. The blue (red) lines indicate the real (imaginary) part of the wavevectors. The full lines are for the chiral Bragg stack with $\gamma_{2}=0.4$, while the dotted lines are for the nonchiral case, $\gamma_{2}=0$. Results are shown for on-axis and oblique incidence of light, respectively. Note the shift of the band edges for oblique incidence only.

To investigate this in more detail, we consider the simpler case where there is negligible index contrast between the layers of the Bragg stack, so that both may be characterized by the same refractive index, $n_{0}$, as illustrated in Fig. 3(a). Several polymers exist with refractive indices close to water; Poly (methyl methacrylate) (PMMA), for example, has a refractive index of $n \simeq 1.49$ and is well suited for photonic crystal manufacturing. ${ }^{9,10}$ One could also imagine index matching by tuning the refractive index of the buffer liquid. Alternatively, microfluidic concepts may be employed to realize a striped pattern with alternating thin laminar-flow layers of a pure buffer fluid (without chiral molecules) and buffer fluid containing chiral molecules. In the low-Reynolds regime, typical for microfluidic architectures, diffusion is the only source of mixing. Well-defined spatial periodic $\gamma$ variations (without any refractive index contrast) should thus in principle be possible by carefully adjusting the flow rate relative to the diffusion rate. ${ }^{11}$

In the case of negligible index contrast, there is no coupling between the two modes of circular polarization and the transmission matrix from medium 1 to medium 2 is characterized by a single scalar transmission coefficient, $t=2 /(1$ $\left.+\cos \theta_{ \pm}^{(2)} / \cos \theta_{ \pm}^{(1)}\right)$, where $\theta_{ \pm}^{(i)}$ are the angles given via the Chiro-Snell's law, $n_{s} \sin \theta_{0}=\left(n_{0} \pm \gamma_{i}\right) \sin \theta_{ \pm}^{(i)}$. ${ }^{12}$ Here, $n_{s}$ is the index of refraction of the surrounding medium, assumed
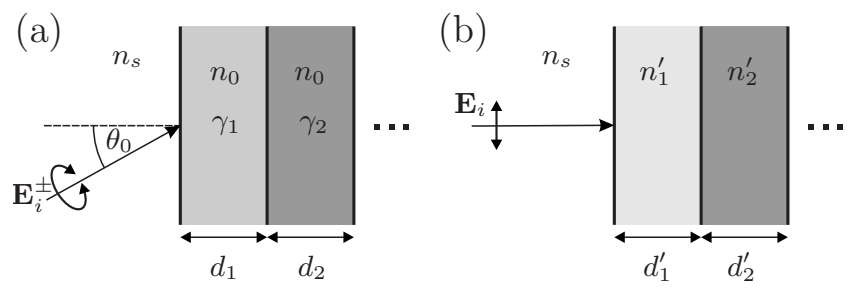

FIG. 3. (a) Chiral Bragg stack with negligible index contrast between layers. The incident light is circularly polarized and at an angle $\theta_{0}$ to the axis of the Bragg stack. This situation can be directly mapped onto (b) a nonchiral Bragg stack with index contrast, where linearly polarized light is incident on axis. $n_{s}=1$ from here on, while $+(-)$ denotes the direction of circular polarization of the incident light, corresponding to right (left) circular polarization. It is evident that this transmission coefficient is of the same form as the one describing on-axis transmission between two nonchiral dielectric layers of index contrast $n_{2}^{\prime} / n_{1}^{\prime}=\cos \theta_{+}^{(2)} / \cos \theta_{+}^{(1)}$. Since the cross-polarization (XP) coupling is negligible, we may thus directly map this problem onto the one illustrated in Fig. 3(b), in which linearly polarized light is incident on an ordinary, nonchiral Bragg stack with layers of refractive indices $n_{1}^{\prime}$ and $n_{2}^{\prime}$. By equating transmission coefficients and phase accumulations in each of the two cases, we arrive at the mapping

$$
\begin{aligned}
& d_{1}^{\prime}=\frac{\Lambda}{1+\gamma_{r} d_{r}}, \\
& d_{2}^{\prime}=\frac{\gamma_{r} d_{r} \Lambda}{1+\gamma_{r} d_{r}}, \\
& n_{i}^{\prime}=\Lambda^{-1}\left[d_{1}\left(n_{0} \pm \gamma_{1}\right)+d_{2}\left(n_{0} \pm \gamma_{2}\right)\right] \cos \theta^{(i)},
\end{aligned}
$$

where $d_{i}$ is the layer thickness of the $i$ th layer of the chiral structure, while $d_{i}^{\prime}$ is the one for the nonchiral structure. Here, we have introduced $\gamma_{r} \equiv\left(n_{0} \pm \gamma_{2}\right) /\left(n_{0} \pm \gamma_{1}\right)$ and $d_{r}$ $\equiv d_{2} / d_{1}$. In this way, the chiral contrast of the zero index contrast case is mapped onto an index contrast of a nonchiral structure.

Using this mapping we may greatly simplify the analysis of the chiral Bragg structure, using terms and results obtained from ordinary index-contrast Bragg structures. First of all, it is clear from the mapping that no band gaps will be present for the structure in the case of on-axis incidence, for which $n_{1}^{\prime}=n_{2}^{\prime}$. Also, we may derive an analytical expression for the first-order shift of the band gap wavelengths as a function of the chiral parameter of either layer. The wavelengths of the band gaps are evaluated using the Bragg condition $m \lambda_{\text {gap }}=2 \Lambda n_{\text {avg }}$, where $n_{\text {avg }}$ is the average refractive index and $m$ is the order of the gap. We find

$$
m \lambda_{\text {gap }}=\sum_{i=1}^{2} 2 d_{i}\left(n_{0} \pm \gamma_{i}\right) \sqrt{1-\frac{n_{s}^{2}}{\left(n_{0} \pm \gamma_{i}\right)^{2}} \sin ^{2} \theta_{0}} .
$$

Fixing $\gamma_{1}$, we find that the sensitivity of the band gaps to the chirality of the second medium is

$$
m \frac{\partial \lambda_{\text {gap }}}{\partial \gamma_{2}}= \pm \frac{2 d_{2}}{\cos \theta_{ \pm}^{(2)}}
$$

where $\cos \theta_{ \pm}^{(2)}=\sqrt{1-\left[n_{s} \sin \theta_{0} /\left(n_{0} \pm \gamma_{2}\right)\right]^{2}}$. There is thus a first-order shift in the band gap positions,

$$
m \Delta \lambda_{\text {gap }} \simeq \pm \Delta \gamma_{2} \frac{2 d_{2}}{\cos \theta},
$$

where $\cos \theta=\sqrt{1-n_{s}^{2} \sin ^{2} \theta_{0} / n_{0}^{2}}$, suggesting that this may be a very sensitive way of measuring chirality. Quite intuitively, the sensitivity increases with the width of the chiral medium. Thus the minimum detectable wavelength shift divided by the width of the chiral layer is ultimately what determines the minimum detectable shift in chirality. As such, simply increasing the width of the chiral layer will increase the sensitivity, so long of course that the concentration of chiral molecules remains the same and that they are still reasonably evenly distributed within the layer. As an example, assuming 

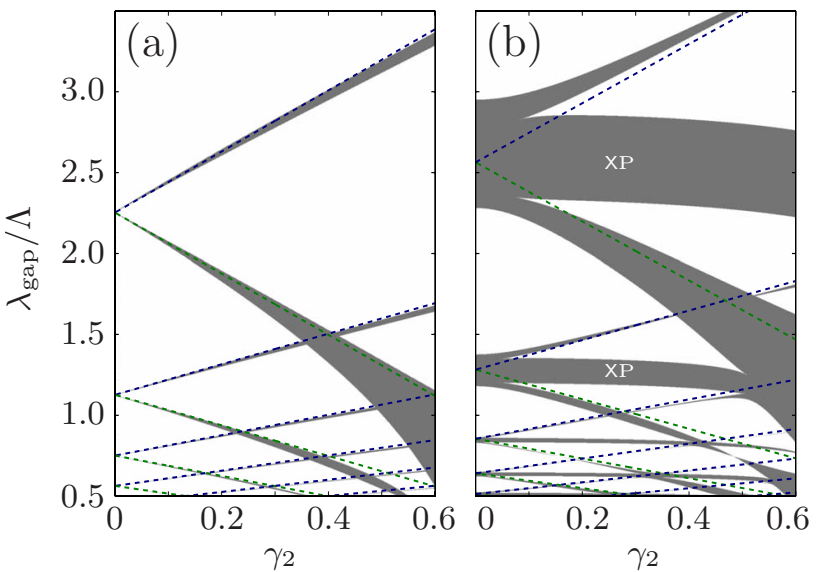

FIG. 4. (Color online) Band gaps for the chiral Bragg stack. The gray areas indicate the regions in which the imaginary part of the wavevector is nonzero, i.e., the band gap regions. Results are for a structure with $d_{1}=0.2 \Lambda$, $d_{2}=0.8 \Lambda, n_{2}=1.33, \gamma_{1}=0$, and oblique incidence, $\theta_{0}=\pi / 4$. (a) No index contrast, i.e., $n_{1}=n_{2}=1.33$. The dashed lines show the analytical expressions Eq. (6) for the first order shift in the central band gap wavelength with the chiral parameter. Blue (green) lines indicate band gaps for right (left) circularly polarized light. (b) Index contrast, $n_{1}=2$. Here, the dashed lines show the approximate analytical results, but with $n_{0}$ replaced by $d_{1} n_{1}+d_{2} n_{2}$. The longest-wavelength XP band gaps are indicated.

a wavelength resolution of $0.1 \mathrm{~nm}$, a layer width of $5 \mu \mathrm{m}$, and setting $\cos \theta \simeq 1$, we obtain a chiral resolution of $\Delta \gamma$ $=10^{-5}$. Interestingly, the sensitivity appears to diverge as $n_{s} \sin \theta_{0} \rightarrow \pm\left(n_{0} \pm \gamma_{2}\right)$. This is, however, related to an effective Brewster's angle and thus coincides with the disappearance of the band gaps, as is also evident from the mapping in Eq. (3c). Also, it is only attainable in the situation of extremely large chirality strengths.

In Fig. 4(a) we show the numerically calculated band gap regions as well as the analytical results based on the first-order expansion in $\gamma_{2}$, for a chiral Bragg stack with no index contrast. The numerical results are based on the solution of the full chiral problem. We find excellent agreement between the analytical and the numerical results. In Fig. 4(b) we show the corresponding results for the case of nonzero index contrast, with $n_{1}=2$. Here, a simple modification of the result in Eq. (6), taking $n_{0} \rightarrow d_{1} n_{1}+d_{2} n_{2}$, gives very good agreement with the full numerics. This shows that the first- order shift in the band gaps is also present in the case of index contrast, and thus the index matching may not be essential for chirality sensing applications. However, as an index contrast is introduced, cross-coupling between the two modes of circular polarization is also introduced, leading to the appearances of XP band gaps, as indicated in the figure. It is evident from the results that these gaps are less sensitive to changes in chirality. From a sensing perspective, this may be problematic, as the XP gap may mask the underlying copolarization $(\mathrm{CP})$ gaps. One possible solution would be to introduce strong chirality in the other medium, i.e., having $\gamma_{1}>0$ in the present case, which will split the CP gaps away from the XP gap.

In conclusion, we have shown how spectral shifts of the photonic band gaps of one-dimensional structured materials may serve as a useful way of measuring chirality. Using a mapping from the case of pure chiral contrast to the case of pure index contrast, we have derived an expression for the first-order shift of the band gaps with chirality. A simple modification of this result is in excellent agreement with full numerics, also in the case of a nonzero index contrast.

This work is financially supported by the Danish Council for Strategic Research through the Strategic Program for Young Researchers (Grant No. 2117-05-0037) as well as the Danish Research Council for Technology and Production Sciences (Grant Nos. 274-07-0080 and 274-07-0379).

${ }^{1}$ L. Chen, Y. J. Zhao, F. Gao, and M. Garland, Appl. Spectrosc. 57, 797 (2003).

${ }^{2}$ A. Ghosh and P. Fischer, Phys. Rev. Lett. 97, 173002 (2006).

${ }^{3}$ J. Pedersen and N. A. Mortensen, Appl. Phys. Lett. 91, 213501 (2007).

${ }^{4}$ F. Jonsson and C. Flytzanis, J. Opt. Soc. Am. B 22, 293 (2005).

${ }^{5}$ E. U. Condon, Rev. Mod. Phys. 9, 432 (1937).

${ }^{6}$ M. P. Silverman, J. Opt. Soc. Am. A 3, 830 (1986).

${ }^{7}$ J. Lekner, Pure Appl. Opt. 5, 417 (1996).

${ }^{8}$ K. Kim, H. Yoo, D. H. Lee, and H. Lim, Waves Random Complex Media 16, 75 (2006).

${ }^{9}$ Y. Oki, T. Yoshiura, Y. Chisaki, and M. Maeda, Appl. Opt. 41, 5030 (2002).

${ }^{10}$ A. Chen, B. Z. Wang, S. J. Chua, O. Wilhelmi, B. M. Shahrain, B. T. Saw, J. R. Kong, and H. O. Moser, Int. J. Nanosci. 5, 559 (2006).

${ }^{11}$ T. M. Squires and S. R. Quake, Rev. Mod. Phys. 77, 977 (2005).

${ }^{12}$ D. L. Jaggard and X. G. Sun, J. Opt. Soc. Am. A 9, 804 (1992). 